\title{
TTR
}

Traduction, terminologie, re?daction

\section{Notes sur une traduction}

\section{Charlotte Melançon}

Volume 12, numéro 2, 2e semestre 1999

Poésie, cognition, traduction II - Autour d'un poème de W. H. Auden

Poetry, Cognition, Translation II — On a Poem by W. H. Auden

URI : https://id.erudit.org/iderudit/037371ar

DOI : https://doi.org/10.7202/037371ar

Aller au sommaire du numéro

\section{Éditeur(s)}

Association canadienne de traductologie

ISSN

0835-8443 (imprimé)

1708-2188 (numérique)

Découvrir la revue

\section{Citer cette note}

Melançon, C. (1999). Notes sur une traduction. TTR, 12(2), 57-65.

https://doi.org/10.7202/037371ar d'utilisation que vous pouvez consulter en ligne.

https://apropos.erudit.org/fr/usagers/politique-dutilisation/ 


\section{Notes sur une traduction}

\section{Charlotte Melançon}

1. Ce que je vous offre est une sorte de journal de bord qui vous présentera, l'une après l'autre, les étapes par lesquelles je suis passée pour essayer de traduire le poème d'Auden. Il ne s'agit pas d'un excès de zèle ou d'honnêteté. Je dois admettre au départ que je n'avais pas gardé un souvenir impérissable de ce poème qui apparaît dans la deuxième partie des Collected Poems publiés par Mendelson en 1976, dans une section intitulée " Five Songs » et qui ne fait aucune allusion au fait que le texte avait d'abord paru dans The Orators. C'est donc dire qu'en commençant ce travail je ne savais même pas si j'arriverais à traduire le poème - ce dont je ne suis pas sûre encore maintenant.

La première lecture m'a laissée, je l'avoue, assez perplexe, d'une part, parce que Auden a écrit dans un nombre incroyable de formes poétiques qui vont de l'élégie à la charade en passant par le psaume et l'oratorio, et que je ne voyais pas à quel genre précis appartiennent ces "Trois compagnons "; d'autre part, parce que Auden écrit généralement en vers réguliers, ce qui pose aux traducteurs des problèmes techniques, de maîtrise de la versification, ou de ce que j'aimerais appeler "l'invention contrôlée ". Il faut dire qu'il existe à notre époque et, en particulier, dans le domaine de la poésie française, une sorte de malentendu à propos du vers libre : un poète moderne est, par définition, celui qui refuse le vers régulier. C'est, bien sûr, une erreur grossière, les plus grands poètes contemporains français l'ayant fait; je cite au hasard Valéry, Apollinaire, Claudel; même un poète surréaliste comme Éluard a écrit des poèmes rimés, à scansion régulière. Aujourd'hui encore Yves Bonnefoy ou Jacques Réda le font, et Jacques Roubaud a écrit des sonnets. Pourquoi donc ce recul des traducteurs devant une technique 
vieille comme le monde et qui appartient à la nature même du poème? On lit parfois que le traducteur ne doit pas « forcer " le vers, qu'essayer de rendre la régularité formelle et rythmique du poème, c'est le tordre ou le défigurer, le faire rentrer dans un carcan qui va nécessairement en déformer le sens. Ce n'est pas tout à fait faux et nous connaissons tous des traducteurs pirouettistes; je pense ici à un traducteur des sonnets de Shakespeare dont parle Jaccottet dans sa préface d'Une lyre à cinq cordes; ce n'est même pas drôle, c'est ridicule. Quoi qu'il en soit, entre l'enjolivure et la re-création il y a loin.

Tout cela pour dire qu'avant même de m'essayer à un premier brouillon, je prends le parti de respecter dans la mesure du possible tous les aspects techniques du poème. Il faut ajouter à cela que Auden luimême n'aimait pas beaucoup le vers libre. Je vous rappelle à tout hasard ces deux paragraphes de "Writing » dans The Dyer's Hand:

[...] lui feront savoir; s'il manque d'autorité, ils deviendront négligents, impertinents, malhonnêtes et ils se soûleront.

"Le poète qui écrit en vers libre " ressemble à Robinson Crusoé sur son île déserte : il doit faire lui-même toute sa cuisine, sa lessive et son reprisage. Sauf dans quelques cas, cette indépendance virile produit quelque chose d'original et d'impressionnant, mais le plus souvent il en résulte de la saleté - des draps sales sur le lit défait et des bouteilles vides sur le plancher poussiéreux. ("Writing », p. 22)

La métaphore de la maison est intéressante : image d'ordre et de propreté, de bon fonctionnement des choses; les " serviteurs " nettoient la place, règlent une certaine ordonnance. Cette maison est en outre une image de l'héritage poétique, un lieu que l'on habite, plein d'outils nécessaires à la vie réussie du poème. Ajoutons que cette métaphore domestique, "rassurante " en quelque sorte, n'a pas empêché Auden de travailler avec Stravinsky ou Britten, ou encore d'écrire ces poèmes qui figurent parmi les plus difficiles de la langue anglaise.

Une première lecture du poème accroche l'œil dès le départ.

a) Ses césures très tranchées révèlent déjà une composition particulière : ces trois paires de noms (propres et communs tout à la fois - il n'y a pas d'articles -) à la première ligne des trois premières strophes sont frappantes; leur reprise dans la dernière ne l'est pas moins. 
Tout cela marque une organisation textuelle singulière. En outre, puisque ces " compagnons " sont groupés par deux, on sait déjà qu'on aura affaire à un débat. Ajoutons à cela que chacun de ces "couples " comportent tous une initiale identique $: \mathrm{r} / \mathrm{r}, \mathrm{f} / \mathrm{f}, \mathrm{h} / \mathrm{h}$.

b) Le poème est entièrement construit sous la forme d'un dialogue comportant une formule semblable : " Said [...] to "

c) Les quatre strophes commencent toutes par un « $O$ ".

d) Il y a une paire de rimes dans chaque strophe : burn/return, pass/grass, trees/disease, reader/fearer, sans compter les assonances.

Voilà d'abord, avant même de comprendre le sens du texte, ce que j'y ai vu et je me suis donc demandée quelle importance jouent dans le poème de Auden tous ces signaux visuels. Si j'avais d'abord entendu le poème, y aurais-je VU les mêmes choses? Chose sûre, c'est que le poème - qui est un dialogue - suppose l'usage de la parole, l'intervention de la voix humaine et que, par ailleurs, étant donné que le poème appartenait d'abord à un recueil qui s'appellait The Orators, il fait appel à la technique oratoire qui possède tout un répertoire de ressources permettant l'insistance, la mise en valeur des mots, bref une diction qui lui est propre. L'agencement visuel du texte n'est donc pas ici indépendant de son élocution. Qu'on l'entende ou qu'on le voie, il existe d'évidence des rapports entre sa double composition typographique et vocale.

PARENTHĖSE : Avant de poursuivre, nécessité de lire le recueil The Orators, voir où se situe ce poème par rapport à l'ensemble. Emprunt à McGill de deux éditions du recueil : la première, de 1932, la troisième, de 1966. "Les trois compagnons » s'appellent dans les deux cas Épilogue, dernier poème donc de l'ouvrage. Lecture plutôt déconcertante; le poème en question n'est pas moins troublant ou mystérieux que l'ensemble. La troisième édition contient toutefois une préface où l'auteur tente de s'expliquer; il croyait avoir écrit un livre sur le culte du héros, il pense maintenant que sa raison d'être était thérapeutique, "visant à exorciser certains fantasmes ". Je reprends ma lecture des Orators en notant toutes les occurrences de mots (par exemple grove, furnace, path, horror, valley, rider, bird, etc.) que l'auteur reprend dans le texte de conclusion; ça pourra peut-être servir. FIN DE LA PARENTHĖSE. 
2. Je commence la traduction proprement dite en dressant une liste des mots accouplés (qu'on pourrait appeler aussi des allégories) qui occupent la seconde partie du premier vers des trois premières strophes.

Je me donne comme principe que le premier étant READER, mot-clé du recueil (p. $85,1^{\text {ère }}$ ode) et premier de la série, il doit me servir en quelque sorte d'étalon ou de mesure pour les cinq autres. Ce READER donc, mot à deux syllabes et se terminant en -eur, aura à porter une charge non seulement sémantique, mais également homophonique, d'autant plus que les cinq autres ont tous la même finale en -eur et comportent également deux syllabes. Ce mot-clé doit jouer à mes yeux et à mon oreille le rôle non seulement d'un " instrument servant à faire fonctionner un mécanisme », selon le premier sens du mot " clé » du dictionnaire, mais aussi de rôle " d'explication, de solution » au problème que pose le poème, sans oublier, bien sûr, son sens musical. READER donne la clé, il est une clé.

Voyons les traductions possibles :

READER : lecteur, liseur, bouquineur, professeur (" lecturer ", assistant adjoint à un professeur de langues vivantes) et, dans son premier sens étymologique en islandais, aviseur; mais aussi, érudit, interprète et traducteur.

Je conserve d'emblée LECTEUR parce qu'il est en français comme en anglais le terme générique, celui qui englobe le plus grand nombre de sens; ce lecteur peut donc être à la fois celui qui lit à haute voix ou celui qui lit seul. Ce « LECTEUR » ici parle.

Voyons la traduction des autres termes:

RIDER : cavalier, écuyer, chasseur, marcheur, éclaireur, galopeur, trotteur; je retiens marcheur, chasseur, trotteur et coureur.

FEARER : peureux, craintif, trouillard, froussard, timide, poltron, trembleur; je retiens trembleur malgré son emploi rare en français moderne (se trouve chez Marcel Achard). Quoi qu'il en soit, « fearer » ne se trouve pas dans tous les dictionnaires d'usage anglais. 
FARER : voyageur, visiteur, pèlerin, baladeur, promeneur, colporteur, chemineau, piéton, passager, randonneur, bourlingueur, flâneur, explorateur, globe-trotter; je retiens flâneur.

HORROR : horreur, effroi, terreur, peur, noirceur, laideur, haine, dégoût; je retiens horreur et terreur.

HEARER : auditeur, ausculteur, guetteur, observateur, veilleur, patrouilleur, explorateur; je retiens guetteur et veilleur.

J'arrive dans chaque cas à conserver un mot de deux syllabes avec une terminaison en -eur, mais je suis absolument incapable de respecter l'initiale de chacun des mots, $\mathrm{R}$ de reader, $\mathrm{R}$ de rider, etc. Perte : dans ces jeux de mots, à quoi le traducteur doit-il accorder sa préférence? À cause des assonances, je préfêre conserver les finales en eur.

Je songe à une autre solution : celui qui lit, celui qui marche, celui qui a peur, celui qui voyage, celui qui fait (ou qui a) peur, celui qui écoute. Je perds mes assonances en -eur et cela risque d'être trop long pour le compte du vers. Je pose ça en attente. Le traducteur, par définition, s'essaie, se hasarde, teste, cherche, se met lui-même à l'épreuve.

Autre question : à quel type de poème appartiennent « Les trois compagnons $»$ ? On ne traduit pas une églogue comme un quatrain du Common Prayers' Bcok. Si Auden se préoccupe presque toujours de donner en sous-titre des indices sur la nature de ses recueils, il faut avouer que celui-ci - An English Study - est plus que vague. Dans son étude sur The Orators, Stan Smith a relevé les genres suivants :

un discours de fin d'année, une discussion, une prière et une litanie, une déclaration, une lettre, un journal, un test de Q.I, avec des figures géométriques, une sextine, un alphabet, des listes, des énigmes, des élégies, des triades gnomiques celtes, une interview, un pamphlet et une conférence, une pasquinade, un récit de rêve et une analyse de rêve, des 
dépêches militaires, des manuels scientifiques, des contrats, des odes pindariques, des rapports de journaux, des psaumes.

Tout cela, en outre, mêle vers et prose. Comme Auden a réinscrit plus tard ce poème dans un groupe de "Cinq chansons ", il est plus que probable que l'Épilogue soit une petite ballade comportant un refrain final. Traduction, donc, assez légère mais également respectueuse de la tonalité du texte pour marquer l'ironie, avec un bon beat. Je mets tout cela en alexandrins.

3. Allons-y. Premiers brouillons que je garde naturellement pour moi : vers de 11 ou de 13 syllabes, césures baroques...

\section{ÉPILOGUE}

« Mais où vas-tu donc?, dit le lecteur au marcheur, Quand fument ses fourneaux cette vallée est traître, Il y traîne un fumier dont les odeurs affolent, Ce trou est une tombe où retournent les maîtres. 》)

« Mais qu'espères-tu, dit le trembleur au flâneur, L'ombre va retarder ton cours vers le passage Et ton œil diligent saura-t-il voir le vide Que tes pas sentiront entre pierre et herbage? »

" Mais quel est cet oiseau? ", dit l'horreur au veilleur, «As-tu vu cette chose entre les nœuds des arbres?

Derrière toi, rapide, une forme se glisse, La tache sur ta peau est un mal exécrable. »

" Hors de cette étude ", - dit le marcheur au lecteur, "Tu n'y verras rien ", - dit le flâneur au trembleur, " On te court après », - dit le veilleur à l'horreur, Alors qu'il les quittait, alors qu'il les quittait.

Dans quelle mesure le traducteur doit-il justifier sa traduction? Est-elle sa propre justification? Il est sûr que certaines traductions sont meilleures que d'autres, plus «entières», dirais-je, parce qu'elles ramassent davantage d'éléments sémantiques et rythmiques, qu'elles tiennent 
compte de toutes ces petites choses qui ajoutent à la densité du poème. Dans le cas de ce poème, ce qui me semble le plus évident, c'est qu'il ne peut pas avoir le même sens si on le remet en contexte, par exemple, dans le recueil d'origine, et c'est le parti que j'ai pris; je regrette évidemment de ne pas avoir eu le temps de le traduire en partant des "Five Songs " d'une autre édition, ce qui aurait sûrement donné un autre résultat. Auden, on le sait, aimait jouer du ciseau et il était certainement conscient de ce fait : l'Épilogue des Orators de 1932 n'est pas tout à fait le même poème que celui des Collected Poems où Auden l'a transformé en " chanson ». Cela a joué, bien sûr, dans ma traduction, d'autant plus que le poème est le dernier de l'ouvrage, qu'il s'oppose en bonne et due forme à un " Prologue » et qu'il ramasse en quelque sorte toute une part de la portée sémantique des textes qui précèdent.

Je vous ai dit qu'en relisant tout le recueil, j'étais allée chercher les termes qui réapparaissent dans l'Épilogue. Or, tous les mots du poème s'échelonnent dans le recueil à l'exception de " midden ", mot dialectal, de " farer " et " hearer " : dans ces deux derniers cas, toutefois, ils apparaissent à maintes reprises sous la forme de " fare » et de " hear ». L'Épilogue répond manifestement à une nécessité, il entend clore le recueil et essaie de résoudre les problèmes que posent l'ensemble. Je n'ai évidemment pas la prétention d'avoir résolu les énigmes du poème ou de cet ouvrage auquel se bute la critique depuis cinquante ans. De l'interprétation freudienne à l'interprétation marxiste ou surréaliste, on a tout tenté. Chose sûre, le recueil est fait pour dérouter et les expressions telles que " practical joke ", " riddle » ou " puzzle » qu'emploie souvent Auden n'y apparaissent pas pour rien. Il y entre une part de jeu et c'est ce qui a " joué » le plus, je dois dire, dans ma traduction; comme l'original, elle devait au fur et à mesure que " j'entrais » dans le texte, mystifier, l'envelopper un peu de ce même mystère. Mais cela ne revenait pas à une dérobade, puisqu'il fallait que ce mystère réponde " le plus justement " possible à celui de l'original.

\section{Exemples :}

Les grands couples antithétiques ou les grandes allégories lecteur/marcheur, trembleur/flâneur, horreur/veilleur - répondent et renvoient de toute évidence à la matière de l'ouvrage. Il me semblerait erroné, notamment, de traduire " rider " par écuyer ou cavalier puisqu'on ne monte pas à cheval dans The Orators, alors qu'on y marche beaucoup. 
J'aurais pu, par ailleurs, traduire " farer " par voyageur, ce qui n'aurait pas gêné le sens, mais j'avais besoin d'un mot de deux syllabes pour rétablir mon antithèse. Cela dit, le flâneur me semblait approprié étant donné « l'errance » de l'esprit de l'auteur; il faut entendre ici le terme non pas dans son sens péjoratif, mais dans celui où Ponge, par exemple, parle de la " promenade professionnelle du poète ". "Veilleur " pour "hearer" est sans doute le plus audacieux des six, mais là encore, puisque Auden l'oppose à " l'horreur ", il me fallait un terme qui contrebalance l'opposition, " veilleur " supposant l'attention, la vigilance ou le contrôle de soi.

Autre exemple: The gap is the grave where the tall return.

Pourquoi " les maîtres "? Cela vient du " Discours de fin d'année " où Auden s'en donne à cœur joie sur le genre d'enseignement " cicéronien », fondé sur l'art oratoire, qui prévalait alors en Angleterre. Il était lui-même enseignant au moment où il a composé son recueil et le but de ce discours (" Vénus va maintenant vous adresser la parole », ou encore, " dépêchons, je ne dois pas rater mon train... ") visait manifestement à tourner en ridicule cette pédagogie qui, selon le poète, se fondait essentiellement sur le pouvoir des maîtres et la peur qu'ils faisaient régner parmi les élèves.

Autre exemple :

La dernière strophe est la plus difficile à traduire parce qu'elle résume le poème qui est lui-même un résumé de l'ouvrage. Le dialogue est, par ailleurs, extrêmement elliptique et comment ramasser en un demivers tout le précédent dialogue?

1. "Hors de cette étude " vient du sous-titre " An English Study " sur lequel Auden joue tout au long de son livre : étude sur les Anglais, sur la langue et la prosodie anglaises, et sur cette pièce où on les étudie.

2. " Tu n'y verras rien » : le " Yours », j'avoue, est plus ambigu, mais il me semble s'appliquer à " looking " plutôt qu'à " footsteps ". Étant donné que ce vers est, comme les autres, un revirement de la strophe auquel il correspond, le « trembleur » autrement dit n'arrivera pas à voir ce vide devant lui, ou encore, de façon symbolique, à aller au-delà 
de sa propre peur. J'ai perdu, hélas, le « never », mais je me rattrappe un peu en ayant recours à l'emploi du futur et de " rien », qui dresse une sorte de mur devant le «trembleur».

3. "On te court après " : pourquoi, en anglais, ce " they " pluriel? À l'exception "d'arbres », toute la troisième strophe ne contient que des mots au singulier : bird, shape, spot, skin, disease. Embetant! $\mathrm{Si}$, par ailleurs, on va chercher dans l'ouvrage tout ce qui a le moindre rapport aux sources de terreur, on n'a que l'embarras du choix : dans sa préface, Auden parlait de thérapie. Il est évident que The Orators porte en grande partie sur son homosexualité et les problèmes qu'elle lui posait. L'ouvrage entier déborde de corbeaux, de faucons et d'aigles, de cauchemars, d'ombres maléfiques, de gouffres, de falaises et d'écueils, de tombes, de maux de toutes sortes. Ce " they " renvoit donc possiblement à toutes ces sources d'angoisse, et c'est la raison pour laquelle je l'ai traduit par ce « on » neutre au lieu de « ils ».

4. Le « il » du dernier vers, qui est-il? J'avais d'abord songé à " elle " puisque c'est " l'horreur » qui termine le vers précédent. Tout compte fait, je pense qu'il s'agit du poète, tout à la fois lecteur et marcheur, trembleur et flâneur, horreur et veilleur, bref toutes ces figures contradictoires qui se trouvent à l'intérieur de l'auteur lui-même. C'est le poète qui s'en va et qui n'écrira plus ce genre d'ouvrage, partagé alors entre le marxisme et les théories freudiennes; le Auden à venir est le grand poète humaniste, celui qui donnera, suite à l'effondrement de l'Europe, une des plus belles définitions de la poésie que je connaisse : un poème est " sorrow controlled by meter " (J. Brodsky, Less than One, New York, Farrar Strauss Giroux, 1986, p. 351).

Charlotte Melançon : 5782, Côte Saint-Antoine, Montréal (Québec) H4A 1S2. Courriel : melancor@ere.umontreal.ca 